\title{
«Très transformés, très emballés et très mauvais pour la santé... mais à faible risque » : exploration des liens entre sécurité des aliments et salubrité des aliments dans les collectivités
}

\author{
Kelsey A. Speed, M. Sc.; Samantha B. Meyer, Ph. D.; Rhona M. Hanning, Ph. D.; Shannon E. Majowicz, Ph. D.
}

Cet article a fait l'objet d'une évaluation par les pairs.

\section{Résumé}

Introduction : L'insécurité alimentaire et les maladies d'origine alimentaire constituent des enjeux importants au Canada, et on peut considérer les mesures de santé publique prises pour les résoudre comme des facteurs qui façonnent l'environnement alimentaire. De nouvelles données probantes allant dans le sens d'une interrelation entre ces deux domaines, l'objectif de cette étude était d'explorer comment, en Colombie-Britannique (Canada), les efforts en matière de sécurité alimentaire des collectivités et les pratiques de salubrité des aliments (ainsi que les problèmes de santé de la population associés) peuvent se recouper, puis d'interpréter ces résultats pour concevoir et construire un environnement alimentaire plus sain.

Méthodologie : Nous avons mené 14 entrevues auprès d'informateurs clés (praticiens des secteurs de la sécurité alimentaire des collectivités et de la salubrité des aliments en Colombie-Britannique) et procédé à une analyse descriptive qualitative pour trouver les points d'intersection entre ces deux secteurs.

Résultats : Les participants ont fait état de quatre modalités de convergence entre les deux secteurs. Ils ont signalé comment les pratiques quotidiennes de leur secteur visant à promouvoir des aliments sûrs ou sains pouvaient être favorisées ou entravées par les activités de l'autre secteur, en partie car les politiques passées, disparates, ne tenaient pas compte des nombreux effets sur la santé en lien avec l'alimentation et car certains types de produits alimentaires, comme les fruits et légumes frais, peuvent être considérés à la fois comme risqués et bénéfiques. Enfin, ils ont souligné que les deux secteurs travaillent à l'atteinte d'un même but, soit celui d'améliorer la santé de la population, même si leur optique se révèle légèrement différente.

Conclusion : La sécurité alimentaire et la salubrité des aliments sont connectées de plusieurs façons, ce qui a une incidence sur les spécificités de l'environnement alimentaire canadien et sur son amélioration. La collaboration entre les divers secteurs de la santé publique en lien avec l'alimentation est nécessaire pour concevoir de nouveaux programmes ou de nouvelles politiques visant à transformer les habitudes alimentaires des Canadiens.

Mots-clés : sécurité alimentaire, salubrité des aliments, pratique en santé publique, politique

\section{Introduction}

L'environnement alimentaire est défini comme l'ensemble des " facteurs physiques, sociaux, économiques, culturels et politiques régissant l'accessibilité, la disponibilité et le caractère adéquat des aliments dans une collectivité ou une région $»^{1}$. Selon cette définition, les mesures prises par les praticiens en santé publique qui modifient la disponibilité et l'accessibilité des aliments peuvent donc être considérées comme des forces qui ont une influence sur l'environnement alimentaire. En outre, les résultats sur une alimentation saine visés par le domaine de la santé publique peuvent être considérés comme des facteurs favorisant la prise de mesures en

\section{Points saillants}

- Les efforts visant à promouvoir la sécurité alimentaire et une alimentation saine peuvent annihiler les efforts déployés pour assurer la salubrité des aliments, et vice versa, même s'ils relèvent tous deux d'un but commun, celui d'améliorer la santé de la population.

- Des politiques disparates et des aliments offrant à la fois des risques et des bienfaits (p. ex. produits agricoles) rendent difficile la mise en place d'améliorations en matière de santé de la population.

- Les mesures conçues pour accroître l'accès à des aliments frais ou limiter les aliments à risque microbien élevé devraient être élaborées conjointement afin d'atténuer les conséquences non voulues.

- Les activités de santé publique relatives aux aliments et à la santé se recoupant de façon non prévue, une collaboration entre ces domaines est nécessaire au moment de la conception des programmes et des politiques visant à transformer les habitudes alimentaires des Canadiens.

santé publique. Dans cette étude, nous avons examiné deux secteurs d'intervention en santé publique liés à l'alimentation - la sécurité alimentaire des collectivités et la salubrité des aliments - ainsi que les problèmes pour la santé de la population qu'ils visent à éliminer (c.-à-d. l'insécurité alimentaire, incluant l'accès à des aliments sains, et les maladies d'origine alimentaire). Les organismes de santé publique, les décideurs et les chercheurs ayant

Rattachement des auteurs :

École de santé publique et de systèmes de santé, Université de Waterloo, Waterloo (Ontario), Canada

Correspondance : Shannon Majowicz, École de santé publique et de systèmes de santé, Université de Waterloo, 200 avenue University Ouest, Waterloo (Ontario) N2L 3G1; tél. : 519-888-4567 poste 31790; courriel : smajowicz@uwaterloo.ca 
toujours étudié séparément ces secteurs, notre étude visait à explorer leurs modalités de recoupement, à la fois dans la pratique en santé publique et sous l'angle de leur influence sur l'environnement alimentaire.

Les activités liées à la sécurité alimentaire visent à assurer que " tous les êtres humains ont, à tout moment, un accès physique et économique à une nourriture suffisante, saine et nutritive leur permettant de satisfaire leurs besoins énergétiques et leurs préférences alimentaires pour mener une vie saine et active $»^{2}$. Cette définition englobe les efforts destinés à améliorer la sécurité alimentaire des collectivités, par exemple les programmes de coupons et les initiatives de la ferme à l'école conçus pour accroître l'accès du public à des aliments frais et sains ${ }^{3,4}$. Les activités liées à la salubrité alimentaire visent à réduire les risques de maladies d'origine alimentaire dans la population, par exemple par des mesures comme l'adoption de lois interdisant les aliments insalubres (p. ex. la Loi sur la salubrité des aliments au Canada ${ }^{5}$, par des enquêtes sur les éclosions et par des rappels d'aliments ${ }^{6,7}$. Malgré la séparation actuelle entre ces activités dans la pratique en santé publique, des données récentes montrent que l'insécurité alimentaire et les maladies d'origine alimentaire ont en amont des déterminants communs. Le faible revenu, par exemple, est un facteur de risque d'insécurité alimentaire et de maladies d'origine alimentaire dans la population, et le changement climatique peut exacerber ces deux situations ${ }^{8}$. Il s'avère aussi, d'après les données, que les mesures de santé publique prises pour résoudre l'un des deux problèmes de santé de la population peuvent avoir une incidence négative et non voulue sur l'autre problème. Par exemple, les programmes communautaires de sécurité alimentaire visant à améliorer l'accès à des aliments sains, comme le Farmer's Market Nutrition Coupon Program ${ }^{3}$, augmentent la consommation de fruits et légumes frais, qui est une source importante d'éclosions de maladies d'origine alimentaire $^{9,10}$. De même, le Meat Inspection Regulation de 2004 (Règlement sur l'inspection des viandes) de la Colombie-Britannique, conçu pour améliorer la salubrité alimentaire, a réduit les capacités de transformation des viandes dans les collectivités éloignées, ce qui a eu pour résultat d'accroître l'insécurité alimentaire ${ }^{11,12}$.
Ces observations semblent indiquer qu'un élément clé, quoique largement méconnu, nécessaire à la compréhension de l'environnement alimentaire canadien consiste à saisir comment les différentes mesures de santé publique dans les secteurs liés à l'alimentation et à la santé peuvent se déployer concrètement de façon non voulue. Même si on sait que les mesures visant à accroître l'accès à des aliments frais et sains (p. ex. interventions portant sur des dépanneurs santé) façonnent l'environnement alimentaire ${ }^{13-16}$ et bien que les activités officielles liées à la salubrité des aliments (p. ex. délivrance de permis, inspection) aient récemment été qualifiées d'outils de politique permettant d'améliorer cet environnement ${ }^{17}$, on manque d'études qui explorent précisément la salubrité des aliments et le risque de maladies d'origine alimentaire dans le contexte d'un environnement alimentaire sain au Canada. Compte tenu de cette lacune, et vu le potentiel d'interrelation entre les maladies d'origine alimentaire, l'insécurité alimentaire et les activités liées à la salubrité des aliments et à la sécurité alimentaire des collectivités (ci-après " sécurité alimentaire »), l'objectif de cette étude était d'explorer, en Colombie-Britannique (Canada), comment les efforts en matière de sécurité alimentaire (et les problèmes d'insécurité alimentaire que l'on cherche à résoudre) et les pratiques de salubrité des aliments (et les maladies d'origine alimentaire que l'on vise à traiter) peuvent se recouper, en recueillant le point de vue de praticiens en santé publique. Nous avons ensuite resitué les résultats de cette étude dans le contexte de l'environnement alimentaire canadien pour proposer des sujets d'études ultérieures.

\section{Méthodologie}

Nous avons réalisé des entrevues auprès d'informateurs clés, soit des personnes sélectionnées car elles œuvraient en santé publique en Colombie-Britannique, dont le travail portait soit sur la sécurité alimentaire soit sur la salubrité des aliments et qui possédaient une expérience de travail avec des praticiens de l'autre secteur. En ce qui concerne les spécialistes en sécurité alimentaire, nous avons inclus les praticiens en sécurité alimentaire et les praticiens en alimentation saine travaillant dans des organismes de santé publique ou dans des organisations communautaires qui visent à accroître l'accès de la population à des aliments sains. Quant aux personnes spécialisées en salubrité des aliments, nous avons inclus les praticiens travaillant dans les organismes de santé publique qui visent à réduire les maladies d'origine alimentaire dans la population. Ces entrevues ont été menées dans le cadre d'une étude plus vaste visant à identifier les obstacles et les facteurs favorables à une collaboration intersectorielle fructueuse. Nous avons élaboré un guide d'entrevue semi-dirigée, qui a permis d'examiner les expériences des participants travaillant avec l'autre secteur, puis nous l'avons testé et révisé en fonction de la rétroaction reçue à propos de la clarté des questions. Nous avons obtenu l'approbation éthique d'un comité d'éthique de la recherche de l'Université de Waterloo (ORE\# 20375).

Les participants ont été recrutés par courriel et ont tous donné leur consentement éclairé verbal au début de l'entrevue téléphonique. Les entrevues, réalisées en janvier et février 2015, ont duré entre une et deux heures et ont été enregistrées. Des notes de terrain ont été également été prises. Les entrevues ont été transcrites, et les transcriptions ont été révisées en fonction des fichiers audio ${ }^{18}$ puis anonymisées. Nous avons supprimé les citations liées à l'expression orale et gênant la fluidité de lecture. Le recrutement des participants s'est poursuivi jusqu'à ce que plus aucun nouveau sujet ne ressorte des entrevues ${ }^{19}$.

Sur les 19 personnes invitées à participer, 14 ont accepté, 1 a refusé et 4 n'ont pas répondu dans les temps. Les 14 participants provenaient de cinq des sept autorités sanitaires de la Colombie-Britannique, de trois organismes provinciaux et de deux organisations non gouvernementales. Ils occupaient des postes de première ligne ou de gestion en sécurité alimentaire $(n=6)$, en salubrité des aliments $(n=5)$ ou dans les deux secteurs $(n=3)$. Tous étaient en milieu ou en fin de carrière. L'échantillon comptait huit femmes et six hommes.

Pour préserver la confidentialité des résultats, les participants sont référencés dans l'article uniquement en fonction du poste ou du secteur. Il était plus facile d'identifier les praticiens en salubrité des aliments par leur poste que ceux en sécurité alimentaire : la majorité d'entre eux étaient des agents d'hygiène du milieu, ainsi que des gestionnaires et des directeurs de ministères de la protection de la santé et de la santé environnementale. À l'opposé, les praticiens en sécurité alimentaire occupaient 
une plus grande variété de postes, que ce soit à la promotion d'une alimentation saine ou à l'amélioration de l'accès aux aliments locaux, comme nutritionnistes communautaires et diététistes en santé publique (ci-après appelés collectivement "diététistes ») ou encore comme chefs de projet.

Nous avons procédé à une analyse descriptive qualitative pour obtenir des exemples de recoupement entre les deux secteurs à partir du discours des participants ${ }^{20}$. Cette analyse a été conduite à l'aide du logiciel ATLAS.ti, version 1.0.50 (282) (ATLAS.ti Scientific Software Development $\mathrm{GmbH}$, Berlin, Allemagne). Nous avons procédé à une analyse des données par induction, conformément à la méthode de Braun et Clarke $^{18}$. Après avoir passé en revue les données, nous avons codé les exemples de recoupement et utilisé ceux-ci pour élaborer des thèmes préliminaires. Nous avons examiné et révisé les thèmes de manière itérative, puis exploré plus à fond chaque thème à l'aide des paramètres et des cas précis décrits par les participants. Nous avons utilisé des notes de travail tout au long du processus de codage pour pouvoir revenir ensuite sur les questions et les réflexions portant sur les données, conformément à la méthode de Birks et ses collègues $^{21}$.

\section{Résultats}

Les participants ont fait état de quatre modalités principales de recoupement entre sécurité alimentaire et salubrité des aliments dans le contexte de la santé publique en Colombie-Britannique. Ils ont expliqué 1) comment les pratiques quotidiennes de leur secteur visant à promouvoir des aliments sûrs ou sains pouvaient être favorisées ou entravées par les activités de l'autre secteur, ils ont relevé 2) que les interrelations pouvaient être compliquées du fait que les politiques, actuellement disparates, ne tiennent compte que d'un effet sur la santé (nutrition ou sécurité alimentaire) et 3 ) et que certains types d'aliments, comme les fruits et légumes frais, peuvent être considérés à la fois comme à risque et comme bénéfiques, selon le point de vue dont on se place. Enfin, ils ont souligné 4) que les deux secteurs travaillent à l'atteinte d'un même but, soit celui d'améliorer la santé de la population, même si leur optique est légèrement différente.

\section{Le point d'intersection entre pratiques spécifiques en santé publique}

Les participants ont décrit comment leurs activités de santé publique ont diversement influé sur, ou ont été influencées par, les efforts de santé publique déployés par l'autre secteur (tableau 1), et notamment comment cette interférence a nui à l'atteinte de leurs objectifs spécifiques en matière de santé publique. Les praticiens en sécurité alimentaire ont par exemple vécu un conflit à propos de la fourniture d'aliments autochtones traditionnels dans des lieux autorisés à fournir ou à servir des aliments au grand public (p. ex. garderies, hôpitaux, salles à manger). Comme l'a expliqué le participant (P) 11 (diététiste), pouvoir servir des aliments traditionnels dans des lieux publics est important pour la sécurité alimentaire :

[...] pour les Premières Nations, la sécurité alimentaire est un enjeu beaucoup plus important que le simple fait d'avoir suffisamment de nourriture. C'est le fait d'avoir des aliments culturellement acceptables. C'est la capacité d'accéder à la terre et à l'eau pour produire ces aliments, et d'avoir des droits sur ces éléments. Être en mesure de servir ces aliments dans un centre de conférence, c'est donc promouvoir, dans un contexte beaucoup plus vaste, les déterminants sociaux de la santé.

Souvent, ces efforts déployés pour fournir des aliments traditionnels dans des lieux autorisés ont été perçus comme freinés par les activités liées à la salubrité des aliments, comme en témoigne P4 (diététiste), qui décrit comment intervient le système HACCP (analyse des risques et maîtrise des points critiques), une approche de la salubrité des aliments qui vise à assurer l'innocuité microbienne des aliments par la mise en place de procédures de contrôle à des étapes importantes de la production alimentaire :

En fait, on soulève un problème important chaque fois qu'on parle d'établissements de soins autochtones, qu'ils soient destinés aux enfants, aux personnes âgées ou aux personnes vivant avec un handicap, peu importe. Ce problème, c'est que [...] il est très difficile d'offrir des aliments traditionnels dans ces établissements, parce que ces derniers veulent assurer la salubrité, et veulent donc s'assurer que les aliments ont suivi un parcours protégé selon la méthode HACCP... [...] Mais les aliments traditionnels ne suivent pas un parcours protégé par HACCP... [...] Cela représente donc un défi de taille, parce que si vous êtes un aîné autochtone, que durant toute votre vie vous avez mangé du poisson en conserve maison ou du poisson fraîchement pêché, que vous vivez maintenant dans un établissement de soins et que vous voulez du poisson, et bien on vous servira des bâtonnets de poisson congelé [XXX] [...] Et pourtant, pour l'instant, aucun établissement n'applique la méthode HACCP de manière à ce qu'on puisse dire que le trajet a été totalement sécuritaire, de l'origine à l'assiette.

Les praticiens en salubrité des aliments ont pris l'exemple d'un conflit vécu lors de leurs activités quotidiennes dans des marchés agricoles locaux, où les partisans de la sécurité alimentaire se sont employés à accroître l'accès à des aliments frais et locaux. Comme l'a expliqué P3 (agent d'hygiène du milieu), avoir déployé des efforts en matière de sécurité alimentaire sans tenir compte des risques de maladies d'origine alimentaire a fait que les agents d'hygiène du milieu — qui sont chargés officiellement de vérifier que les aliments vendus au public sont salubres - se sont retrouvés dans une position où ils ont dû réagir :

[Le groupe responsable de la santé de la population] était en train de dresser une liste des fournisseurs d'aliments locaux... Ils sont arrivés en coup de vent et inscrivaient tout le monde sur la liste, recueillant les noms et les numéros des endroits où on peut acheter, " tout ce qu'on veut ». Le problème, c'est que parmi ce « tout ce qu'on veut » sur la liste figurait notamment de la viande non inspectée. [...] Un jour, les inspecteurs des viandes sont arrivés. Quelqu'un cuisinait des pierogis destinés à la vente et quelqu'un d'autre fabriquait du fromage de chèvre fait de lait non inspecté. Donc, certains problèmes de santé assez graves [...] dans mon esprit. Il n'y avait aucune communication, il n'y avait que des bonnes idées mises en pratique mais 
TABLEAU 1

Exemples de situations vécues par les praticiens en santé publique de la Colombie-Britannique où il y a croisement entre les secteurs de la sécurité alimentaire et de la salubrité des aliments

\begin{tabular}{|c|c|}
\hline Situation & Exemple de citation \\
\hline $\begin{array}{l}\text { Fourniture d'aliments frais, } \\
\text { sains et locaux dans les écoles }\end{array}$ & $\begin{array}{l}\text { Donc, du point de vue de l'agent d'hygiène du milieu, il s'agit surtout - il s'occupe de salubrité des aliments - souvent, les } \\
\text { aliments qui sont sûrs, plus sûrs, sont souvent des aliments emballés et des aliments transformés, ce qui n'est pas conciliable } \\
\text { avec certains aliments que nous essayons de promouvoir dans les écoles. [P10, diététiste] }\end{array}$ \\
\hline $\begin{array}{l}\text { Fourniture d'aliments sains } \\
\text { dans les garderies }\end{array}$ & $\begin{array}{l}\text { Vous pouvez obtenir cette liste d'aliments à faible risque... ils sont hautement emballés, très transformés et très mauvais à la } \\
\text { santé. Mais ils présentent un faible risque, donc il peut y avoir des embûches, mettons, si vous travaillez avec un établissement } \\
\text { préscolaire ou une garderie. Vous voulez que les enfants aient accès à des aliments sains, mais vous vous présentez avec les } \\
\text { mauvaises lignes directrices lorsque vous dites : « Vous ne pouvez pas avoir ces aliments. » Il faut dire : «Vous devez nous } \\
\text { montrer que vos aliments sont correctement préparés. » [P13, responsable de la sécurité alimentaire] }\end{array}$ \\
\hline $\begin{array}{l}\text { Fourniture d'aliments locaux } \\
\text { dans les hôpitaux }\end{array}$ & $\begin{array}{l}\text { On parle de fourniture d'aliments locaux dans les cuisines des hôpitaux parce que ces derniers sont de grands acheteurs } \\
\text { d'aliments. On se dit : « Eh bien, peut-être que nous pouvons demander au fournisseur local de fournir les viandes pour les } \\
\text { produits qu'on sert à l'hôpital, cela serait tout un marché à conquérir », puis vous pouvez penser : « D'accord, mais ont-ils } \\
\text { vraiment l'infrastructure pour soutenir tout ça de manière constante, et peuvent-ils le faire en toute sécurité? »... Je crois que } \\
\text { l'un des arouments employés contre la fourniture locale d'aliments, c'est que les hôpitaux ont besoin d'un grand volume } \\
\text { d'aliments très uniformes qui ne nécessitent pas beaucoup de transformation... La salubrité, c'est autre chose. Ont-ils les } \\
\text { mécanismes requis en place? Vous pensez aux fournisseurs de produits frais. Suivent-ils le programme de salubrité des aliments à } \\
\text { la ferme? Sont-ils conformes aux bonnes pratiques agricoles? Disposent-ils des infrastructures en place pour pouvoir assurer, } \\
\text { j'imagine, la qualité de l'aliment et rassurer les utilisateurs que la qualité est suffisamment élevée et qu'ils n'ont pas à s'inquiéter } \\
\text { du risque lié à la salubrité lorsqu'ils acceptent le produit? [P6, gestionnaire, protection de la santé/santé environnementale] }\end{array}$ \\
\hline $\begin{array}{l}\text { Fourniture d'aliments locaux } \\
\text { dans les marchés agricoles }\end{array}$ & $\begin{array}{l}\text { Nous n'étions pas satisfaits avec les produits dangereux du marché agricole et nous voulions plus d'étiquetage sur les produits } \\
\text { en conserve, et ce genre de choses ne faisait pas partie des tâches [du groupe responsable de la sécurité alimentaire/santé de } \\
\text { la population]. Ils ne faisaient qu'exercer des pressions pour vendre certains aliments locaux. [P3, agent d'hygiène du milieu] }\end{array}$ \\
\hline $\begin{array}{l}\text { Promotion des jardins } \\
\text { communautaires }\end{array}$ & $\begin{array}{l}\text { Je veux dire, j'utiliserais les jardins communautaires dès maintenant, une étude est en cours à l'UBC. Vous avez probablement } \\
\text { lu au sujet de la contamination du sol et du plomb, des concentrations élevées de plomb dans certaines régions de Vancouver } \\
\text { où se trouvent des jardins communautaires. Donc, bien entendu, c'est un énorme problème. Nous ne voulons pas que les gens } \\
\text { soient intoxiqués par le plomb, mais nous n'en discutons pas sous l'angle de la sécurité alimentaire. Il faut peut-être tout } \\
\text { fermer et ne peut plus avoir de jardins communautaires dans la ville de Vancouver, mais, ce n'est pas une bonne nouvelle. [P7, } \\
\text { gestionnaire, sécurité alimentaire] }\end{array}$ \\
\hline
\end{tabular}

Soutien à l'accès à des produits agroalimentaires locaux

[...] mais les gens se disent, j'imagine que c'était l'attitude du genre : « Eh bien, si ça pousse juste l'autre côté de la rue, et qu'il s'agit d'une petite ferme d'une acre, alors ça doit être bon pour nous » et sur le plan de l'agriculture, cela semble merveilleux en effet, et ça serait génial, mais pas nécessairement, et nous ne pourrions pas prendre ce risque, nourrir les enfants des autres. [P9, responsable de projet en sécurité alimentaire]

Mise en place de mesures de salubrité des aliments par des règlements sur les viandes locales

Il faut reconnaître que dans certaines de nos régions rurales plus éloignées, il n'était pas possible de créer un abattoir détenant un permis provincial. On a donc instauré un permis d'abattage à la ferme. Des permis de classe $\mathrm{D}$ et $\mathrm{E}$ sont offerts dans ces régions rurales éloignées, des permis de classe E sont disponibles hors de ces emplacements, ainsi qu'une étude de faisabilité, parce que si vous pouvez amener votre animal à un abattoir, on préfère cette option en raison des normes de salubrité des aliments qui existent à l'abattoir... C'était donc plus ou moins une réponse, reconnaissant que nous voulions continuer de soutenir les aliments locaux, mais que nous souhaitions quand même avoir des normes en place. Parce que nous voulons, bien entendu, nous assurer que tous les BritannoColombiens ont accès à de la viande locale salubre, n'est-ce pas? [P8, gestionnaire, protection de la santé/santé environnementale]

Dons d'aliments à des banques alimentaires et par les cuisines communautaires

Nous considérons actuellement assez souvent les fruits et légumes frais comme l'une des catégories d'aliments les plus risquées [...] en fonction uniquement du nombre d'éclosions survenues au cours des quelque dix dernières années. Les produits frais sont souvent mis en cause dans les éclosions, alors que c'est certainement l'un des types d'aliments que vous voulez voir à une soupe populaire ou dans une banque alimentaire, ou dans un don alimentaire, et qui sont assurément plus sains que des produits comme [...] un Kraft Dinner. Par conséquent, je crois qu'il faut tenir compte de cet aspect, qu'il faut prendre certaines précautions de manutention et qu'il y a des limites à ce qu'il est possible de faire de façon sécuritaire ou non, et cela doit également être pris en compte. [P6, gestionnaire, protection de la santé/santé environnementale]

Soutien à l'utilisation de viande de gibier abattu

Par exemple, avec la viande de gibier abattu, nous recevions des demandes de la part de municipalités ou de districts régionaux du genre : «Nous avons tous ces cerfs abattus et ce serait bien si nous pouvions les transformer d'une façon ou d'une autre et les donner à la banque alimentaire locale ou aux gens des Premières Nations ou à qui que ce soit d'autre. » Nous avons répondu : «Eh bien, oui, ce serait une bonne idée, car ce sont des aliments de bonne qualité. Travaillons donc ensemble pour s'assurer que tout est fait de façon sécuritaire, pour que personne ne tombe malade en consommant ce produit. » [P1, spécialiste de la salubrité des aliments]

Soutien à l'accès aux dons d'aliments sains et locaux par des projets de cueillette

Amélioration de la santé des nouvelles mères et des jeunes enfants
Les programmes de nutrition communautaires organisent des projets de cueillette dans la région [XXX]. II reste beaucoup d'arbres fruitiers à la fin de l'année, donc nous avons travaillé à leur fournir certains conseils sur la salubrité des aliments tout au long du projet de cueillette. [P6, gestionnaire, protection de la santé/santé environnementale]

Ces jours-ci dans le domaine de la santé publique, on organise beaucoup d'ateliers sur l'allaitement à l'intention des nouvelles mères. Nous y accueillons d'autres groupes pour parler de salubrité des aliments, de désinfection, d'hygiène personnelle dans la maison, en particulier s'il y a des animaux de compagnie, de jouets et d'infections qui peuvent survenir à la maison, et comment les prévenir. En présentant des sujets comme le contrôle des infections, les ateliers ont permis d'attirer les gens responsables de la salubrité des aliments, ceux responsables de la sécurité alimentaire et ceux œuvrant dans le domaine de I'alimentation saine, bref, une grande diversité d'intervenants. [P5, agent d'hygiène du milieu] 
sans aucune collaboration ou même demande auprès de nous, donc lorsque nous en avons entendu parler, notre réaction a été de dire : « non, c'est terminé, vous ne pouvez pas faire cela ». Et, bien sûr, c'est la guerre [rires].

\section{Les répercussions des politiques ne tenant compte que d'un seul effet sur l'alimentation ou la santé}

Lors des discussions sur les conflits opposant les tentatives en sécurité alimentaire et celles en salubrité des aliments, les participants ont souligné comment cette situation était en partie due aux politiques et règlements disparates qui n'ont jamais tenu compte des autres effets sur l'alimentation ou la santé dans leur élaboration et leur mise en œuvre. Par exemple, P12 (diététiste) explique comment des lignes directrices comme celles en matière de salubrité des aliments suivies dans le cadre de FOODSAFE, un programme de formation des préposés à la manutention des aliments de la Colombie-Britannique ${ }^{22}$, peuvent causer des problèmes pour les établissements préscolaires qui servent de la nourriture aux enfants :

Nous avons toujours souhaité, par notre programme, le programme de nutrition communautaire, travailler davantage avec les enfants d'âge préscolaire. Nous encourageons et favorisons l'alimentation saine dans ces secteurs, ces installations, mais nous avons constaté que les règlements [sur la salubrité des aliments] jouaient presque en notre défaveur. [...] D’un côté, les titulaires de permis et les fournisseurs de services de garde se faisaient dire haut et fort que le programme FOODSAFE était nécessaire, à un point tel que, selon moi, même si le produit venait dans un emballage de plastique, du genre qui crépite quand on le froisse, on pouvait quand même le servir, car il était certifié FOODSAFE. Puis, de l'autre côté, les nutritionnistes déclaraient " eh bien, nous voulons des aliments sains, qui sont des aliments frais », et je crois qu'ils étaient d'une certaine manière limités dans ce qu'ils pouvaient faire.

P12 poursuit en expliquant que, souvent, les règlements actuels en matière de salubrité des aliments ne tiennent pas compte des répercussions possibles sur l'alimentation saine :

[...] les agents d'hygiène du milieu sont assujettis au Règlement sur les locaux destinés aux aliments. [...] Et les mesures prises par eux et par les agents de délivrance des permis, et nos propres documents, n'offraient pas tout le soutien nécessaire en ce qui concerne l'alimentation saine. [...] Nos exigences en matière de salubrité des aliments à l'intention des fournisseurs de services de garde, des garderies agréées, étaient très fortement orientées vers la salubrité des aliments, sans égard à l'alimentation saine.

Dans certains cas, le décalage entre la politique et son application était implicite dans les déclarations des participants, par exemple, dans la façon dont P13 (responsable de la sécurité alimentaire) parle du Guide alimentaire canadien, la ligne directrice fondamentale dans la province, mais qui ne tient pas compte du risque d'infection à $E$. coli (responsable d'environ 33000 intoxications au Canada chaque année ${ }^{23}$ ) ni des règlements en matière de salubrité des aliments conçus pour minimiser ce risque :

Le Guide alimentaire canadien est essentiellement une ligne directrice nationale [...] Pour l'alimentation saine au Canada, et dans la province, nous utilisons ce guide comme outil et tout le monde s'efforce de faire de meilleurs choix nutritionnels [...] On ne peut donc pas le balayer du revers de la main. On ne peut pas dire que les enfants ne doivent pas manger de salades, parce que c'est dangereux [...] On ne peut pas bannir les hamburgers des établissements préscolaires, n'est-ce pas [rires] à cause du risque d'infection à $E$. coli $[\ldots]$ non.

Au-delà des règlements et des lignes directrices en tant que tels, certains participants ont discuté de la façon dont les différentes interprétations des règlements sur la salubrité des aliments pouvaient avoir des effets négatifs sur la sécurité alimentaire et l'alimentation saine, comme en témoigne P6 (gestionnaire, protection de la santé/ santé environnementale) :

Je crois que pour beaucoup de gens, oui, une lumière s'allume, ils se disent : «Oui, cela a du sens, ce n'est pas si grave, laissez-les faire ", puis d'autres disent : " Mais non, ce n'est pas conforme à la loi », donc, pour certains employés, cela dépend vraiment de leur point de vue sur la manière d'interpréter les lois et dans quelle mesure ils croient avoir le pouvoir de faire respecter l'esprit de la loi, de faire ce qui est probablement la bonne chose à faire.

Les participants ont également expliqué comment réinterpréter les règlements existants peut soutenir à la fois les objectifs en matière de salubrité des aliments et en matière de sécurité alimentaire. À titre d'exemple, P12 (diététiste) a souligné que le Child Care Licensing Regulation ${ }^{24}$ de 2007 appuie en fait autant la sécurité alimentaire que la salubrité des aliments dans les garderies :

[...] Là où il y a eu, selon moi, une certaine adhésion, également, en protection de la santé, c'était le fait que réaliser ces activités alimentaires avec les enfants respecterait réellement certains énoncés ou exigences du Child Care Licensing Regulation. Parce que nous avons consulté le Child Care Licensing Regulation, qui prévoit que - où est-ce indiqué? [traduction] « le titulaire d'un permis doit établir un programme de formation et de mise en pratique des règles de santé et d'hygiène ». Il s'agit en fait de l'article 46... [rires]... du Child Care Licensing Regulation. Nous avons donc fait valoir que les expériences de découverte et de préparation des aliments que nous offrons constituent des occasions pratiques idéales pour sensibiliser les enfants à l'hygiène, à la santé, à la salubrité des aliments et au lavage des mains. Donc, c'était un des arguments, puis il y avait également l'article 48 au complet, Nutrition, qui prévoit pour un enfant, [traduction] " que le titulaire d'un permis doit s'assurer que chaque enfant consomme des aliments et des boissons sains selon le Guide alimentaire canadien » et un tas de choses, n'est-ce pas, et puis nous avons affirmé que la pratique exemplaire consistait à exposer les enfants à diverses expériences alimentaires et à une variété d'aliments sains, qui sont frais et très peu transformés, et que les fournisseurs de services de garde étaient limités aux 
aliments préemballés pour éviter le processus d'approbation. Ils allaient compromettre la qualité nutritionnelle. La qualité nutritionnelle était un élément important du Child Care Licensing Regulation, donc, oui, pour ces deux raisons, dans ce règlement, nous l'avons en quelque sorte retourné en disant : "Ces modifications vous aident vraiment à respecter le règlement ».

P6 (gestionnaire, protection de la santé/ santé environnementale) a également abordé la question de la réinterprétation lors de discussions sur des lignes directrices élaborées pour interpréter la législation sur la salubrité des aliments d'une manière qui soutienne également certaines initiatives en matière de sécurité alimentaire et d'alimentation saine :

Et, pour moi, ce que je constate vraiment dans bien des initiatives de sécurité alimentaire, c'est que les employés doivent en quelque sorte avoir la permission d'aller de l'avant et de tenir compte de ces éléments. Une ou deux choses viennent donc en tête, c'est-à-dire, oui, ils veulent savoir qu'ils ne seront pas pénalisés s'ils approuvent quelque chose qu'ils n'auraient peut-être pas approuvé s'ils avaient suivi la loi à la lettre, mais en plus, il y a une certaine uniformité dans le fait que si vous donnez à quelqu'un l'occasion de faire ce genre de chose, vous pourriez être perçu comme étant un peu laxiste avec la loi, mais s'il existe une ligne directrice pour l'appuyer, ou tout autre document mentionnant qu'il y a eu un précédent, alors " oui, vous pouvez autoriser ceci et ceci et ceci dans ce type d'établissement ». Cette attitude leur donne en quelque sorte l'autorisation d'aller de l'avant et de permettre un adoucissement de cette interprétation rigoureuse de la loi.

\section{L'impact du produit alimentaire : ce qui est sain n'est pas toujours salubre et ce qui est salubre n'est pas toujours sain}

L'essentiel du conflit décrit par les participants sur le plan pratique et stratégique relevait du fait que le risque de maladie d'origine alimentaire peut être plus élevé avec les aliments frais et sains que l'on promeut en matière de sécurité alimentaire, et que les aliments présentant un faible risque en matière de salubrité sont souvent préemballés et transformés, donc moins sains et nutritifs. Les participants ont surtout comparé les fruits et légumes frais et les aliments préemballés et transformés ou les aliments "dans un emballage de plastique, du genre qui crépite quand on le froisse » (P12, diététiste). Par exemple, P6 (gestionnaire, protection de la santé/santé environnementale) a comparé les fruits et légumes frais au Kraft Dinner lors d'une discussion sur les dons de nourriture faits par exemple aux banques alimentaires, décrivant les risques que présentent ces deux types d'aliments :

Nous considérons actuellement assez souvent les fruits et légumes frais comme l'une des catégories d'aliments les plus risquées [...] en fonction uniquement $\mathrm{du}$ nombre d'éclosions survenues au cours des quelque dix dernières années. Les produits frais sont souvent mis en cause dans les éclosions, alors que c'est certainement l'un des types d'aliments que vous voulez voir à une soupe populaire ou dans une banque alimentaire, ou dans un don alimentaire, et qui sont assurément plus sains que des produits comme [...] un Kraft Dinner. Par conséquent, je crois qu'il faut tenir compte de cet aspect, qu'il faut prendre certaines précautions de manutention et qu'il y a des limites à ce qu'il est possible de faire de façon sécuritaire ou non, et cela doit également être pris en compte.

De même, P10 (diététiste) a noté que les aliments dont le risque de maladies d'origine alimentaire est minime ne sont pas toujours sains :

[Je] pense à un document autrefois en vigueur - je crois bien qu'il l'est encore, parce que cette initiative n'est pas encore terminée -, une liste des "aliments sûrs que vous pouvez préparer à l'école". Je crois que nous avons encore un document de Health Link BC sur le programme FOODSAFE qui mentionne que "les pains et pâtisseries, riches en sucre, ou quelque chose comme ça, sont plus sûrs que de préparer des légumes".

Même si l'idée de comparer les fruits et légumes frais et les aliments préemballés s'est révélée prédominante, d'autres types d'aliments ont été mentionnés dans le contexte des liens entre salubrité des aliments et sécurité alimentaire. À titre d'exemple, P6 (gestionnaire, protection de la santé/ santé environnementale) a décrit comment les aliments potentiellement dangereux du point de vue de la salubrité des aliments, comme la viande, les produits laitiers et les œufs, comptent également parmi les aliments les plus nutritifs :

Malheureusement, la législation n'aborde pas vraiment ce type précis d'aliments. Elle traite un peu des aliments potentiellement dangereux, cela a donc toujours été un peu une ligne de démarcation et, malheureusement, beaucoup d'aliments potentiellement dangereux font également partie des aliments les plus nutritifs, donc [...] il y a les produits laitiers, et lorsque vous avez des produits de la viande et des œufs, par exemple, il existe un niveau de risque élevé généralement associé qui s'y rattache, mais à la condition que ce risque découle d'une mauvaise manutention en cours de route.

Dans le même ordre d'idées, P11 (diététiste) a expliqué comment les aliments avantageux sur le plan de la sécurité alimentaire, comme les aliments traditionnels préparés par la population, peuvent présenter un risque de maladies d'origine alimentaire :

[...] du point de vue des Premières Nations, notre environnement a vraiment changé. Les risques de maladies d'origine alimentaire sont nettement plus nombreux que jamais auparavant, et nos méthodes changent également un peu, ce qui augmente le risque. On a qu'à penser au poisson ou au gibier. Certaines personnes aiment - surtout dans le cas du poisson -, certains se tournent vers la mise en conserve ou en pot du poisson... C'est très courant dans les communautés des Premières Nations de le faire dans un bainmarie d'eau bouillante, ce qui n'est pas conforme à la norme de traitement relative à la salubrité des aliments. La norme est la mise en conserve à l'autoclave, parce qu'il est possible d'élever le produit à la température nécessaire pour tuer les 
spores potentielles. C'est ce que nous voulons. Sinon, le risque, en fait, c'est la mort.

Outre les exemples susmentionnés, un participant (P13, responsable de la sécurité alimentaire) a décrit une situation où, dans le cadre de discussions sur le problème des préparations pour nourrissons expirées, les objectifs de sécurité alimentaire et de salubrité des aliments visant à réduire les risques pour la santé étaient conciliables :

[...] avec les préparations pour nourrissons et les aliments pour bébés, le "meilleur avant telle date ", et j'étais très préoccupé par la rancidité... dans les préparations et, bien entendu, il peut s'agir d'une discussion sur la salubrité des aliments, mais il s'agit également d'une discussion très importante sur la nutrition, n'est-ce pas... parce que les acides gras essentiels à longue chaîne, si ils deviennent rances, c'est tout un problème... C'est aussi un problème de nutrition vraiment important. La rancidité n'est donc pas uniquement un risque de toxicité, c'est aussi une composante nutritionnelle.

\section{Reconnaître que pour les deux secteurs, "l'objectif ultime est la meilleure santé possible »}

Globalement, les participants ont parlé de l'importance d'adopter une vue d'ensemble à propos du lien entre les aliments et la santé de la population. Par exemple, P6 (gestionnaire, protection de la santé/santé environnementale) a expliqué que lorsqu'on travaille à améliorer la santé de la population, il importe de voir au-delà de son propre secteur afin de reconnaître le rôle des autres effets sur l'alimentation et la santé :

Je pense qu'on comprend dans une certaine mesure que les aliments ne se résument pas au volet salubrité, qu'il y a beaucoup plus que cela en ce qui concerne les avantages pour la santé publique, et je crois que si vous examinez les déterminants de la santé, et quiconque ayant travaillé dans ce domaine voit clairement que la salubrité des aliments en est une composante mais qu'il y en a beaucoup d'autres, et que bien d'autres caractéristiques des aliments influenceront favorablement la santé publique, donc, qu'il s'agisse de nutrition ou de sécurité alimentaire, des choses peuvent se produire avec les aliments et il faut en être conscient.

De plus, comme P4 (diététiste) l'a fait remarquer, la nourriture joue un rôle prépondérant dans la santé, un rôle qui dépasse largement l'acte physique de se nourrir :

Et la population locale avec laquelle je travaille, avec laquelle nous travaillons tous ensemble, et on m'a entendu expliquer en détail [rires] ce type de problèmes, que la nourriture n'est pas que la nourriture, c'est la culture et [rires] cela va au-delà de la satiété.

Cependant, même s'ils ont noté comment les activités et les politiques relatives à la sécurité alimentaire et à la salubrité des aliments peuvent entrer en contradiction, la plupart des participants étaient conscients que les deux secteurs jouent un rôle important dans l'amélioration de la santé de la population. Par exemple, P1 (spécialiste de la salubrité des aliments) a fait valoir que les deux secteurs accordent de l'importance aux effets sur la santé découlant de la salubrité des aliments : "Dans la plupart des cas, ces secteurs souhaitent voir, comme vous, le même résultat, c'est-à-dire des aliments sûrs. Autrement dit, personne ne souhaite rendre les gens malades. "De même, P10 (diététiste) a souligné qu'un des objectifs de la sécurité alimentaire est d'inculquer de saines habitudes à long terme auprès de la population, et que la salubrité des aliments est souvent intégrée à cet objectif :

Pour ce qui est des garderies et des écoles, c'est le moment où les enfants prennent des habitudes alimentaires qui, espérons-le, serviront de fondement tout au long de leur vie. Dans ce cas, nous voulons donc à la fois des aliments sains et sûrs.

De plus, les participants reconnaissent que la salubrité des aliments est souvent considérée comme un volet important de la sécurité alimentaire, comme en témoigne P1 (spécialiste de la salubrité des aliments) :

[...] Toute l'idée de la sécuritaire alimentaire, vous savez, des aliments bons et nutritifs pour tous, ou l'accès à ceux-ci, mais des aliments bons, nutritifs et sains [...] je crois vraiment que c'est absolument lié à notre thème très central, qui est tout aussi important que la nutrition.

Enfin, pour les participants, le but fondamental des secteurs de la sécurité alimentaire et de la salubrité des aliments est bien d'améliorer la santé de la population, comme l'a mentionné P12 (diététiste) lors de discussions sur les aliments dans les garderies :

Le message que nous essayons de communiquer ressemble à l'objectif ultime : nous visons la meilleure santé possible pour les enfants pris en charge. Cela comprend dans l'immédiat la santé et la salubrité, mais aussi la santé tout au long de la vie, sans oublier les effets des maladies chroniques, et la proportion de la population qui sera touchée par celles-ci à cause de mauvaises habitudes alimentaires et de vie, par rapport au risque immédiat lié à la salubrité des aliments [...] Et essayer d'établir un équilibre, car ces deux volets sont vraiment importants.

\section{Analyse}

Dans cette étude, nous avons cherché à savoir comment se recoupent le secteur de la sécurité alimentaire des collectivités et celui de la salubrité des aliments, dans le contexte de la pratique en santé publique en Colombie-Britannique. Les participants ont révélé comment les pratiques quotidiennes de leur secteur, qui visent à améliorer soit l'accès de la population à des aliments sains soit la salubrité des aliments consommés par le public, pouvaient être favorisées ou entravées par les activités de l'autre secteur, notamment en raison de politiques disparates qui ne tiennent pas compte des nombreux effets sur la santé associés aux aliments. Ils ont en outre déterminé comment certains types de produits alimentaires, comme les fruits et légumes frais, peuvent comporter à la fois des risques et des avantages pour la santé de la population. Malgré ces tensions, les participants ont bien vu que les deux secteurs s'employaient à atteindre le même objectif général, soit l'amélioration de la santé de la population, même si leurs perspectives étaient légèrement différentes, une attitude qui leur a permis de collaborer avec l'autre secteur malgré les difficultés 
auxquelles ils étaient confrontés. Ces résultats conduisent à prendre en compte plusieurs points pour concevoir dans l'avenir l'environnement alimentaire au Canada ainsi que pour prendre des mesures visant à l'améliorer.

D'abord, lorsqu'il s'agit de créer un environnement alimentaire plus sain, la mobilisation des praticiens en santé publique ne doit pas s'arrêter à la mise à contribution des gens du domaine de la nutrition, de l'alimentation saine et de la sécurité alimentaire, mais doit aussi inclure les responsables de la salubrité des aliments. Même si tenir compte de la salubrité alimentaire au moment d'évaluer ou d'agir afin d'améliorer un environnement alimentaire a déjà été suggéré ${ }^{17,25}$, notre étude montre dans quelle mesure la collaboration avec des praticiens en salubrité des aliments (ou son absence ) peut avoir une incidence sur les efforts déployés en santé de la population en vue d'améliorer l'environnement nutritionnel de la collectivité. Dans cette étude, les participants ont fourni de nombreux exemples de la manière dont le manque d'engagement entre les secteurs liés à l'alimentation a compliqué leur mandat de fournir des aliments sains et sûrs aux collectivités concernées. Les travaux de Martin et Perkins ont révélé des tensions entre les praticiens en salubrité des aliments et ceux du domaine de la sécurité alimentaire dans bien des provinces canadiennes ${ }^{26}$, ce qui donne à penser que les conclusions présentées ici pourraient s'appliquer ailleurs qu'en Colombie-Britannique. Cela implique de réaliser des travaux supplémentaires pour déterminer comment mieux soutenir la collaboration entre praticiens de ces secteurs.

Au-delà des actions individuelles menées par les praticiens en santé publique, nos conclusions donnent à penser également que les politiques provinciales et fédérales relatives aux aliments et à la santé devraient tenir compte des effets et des influences possibles sur la santé au-delà du résultat concret visé. Cette idée a déjà été proposée $^{8}$, et notre étude montre bien que des politiques disparates peuvent réduire l'efficacité des mesures prises par les praticiens de première ligne, qui doivent, lors de la mise en œuvre des programmes, naviguer en évitant les écueils des divergences entre milieu stratégique et milieu législatif. Les lignes directrices officielles peuvent faciliter cette navigation, particulièrement lorsqu'elles sont le fruit d'une collaboration. Par exemple, la Food Donor Encouragement Act de 1997 de la Colombie-Britannique (qui dégage de toute responsabilité les donateurs agissant de bonne foi si le don de nourriture a des répercussions négatives sur la santée ${ }^{7}$ ) peut agir en contradiction avec les normes traditionnelles en matière de salubrité des aliments, créant ainsi des situations dans lesquelles les praticiens en santé publique peuvent se retrouver avec des objectifs entrant en contradiction (p. ex. accroitre les dons d'aliments frais ou mettre en application les normes de salubrité des aliments), particulièrement pour certains aliments comme les fruits et légumes frais et les aliments transformés, comme l'ont fait remarquer nos participants. En 2016, la Colombie-Britannique a établi des lignes directrices élaborées conjointement par les responsables de la salubrité des aliments et les représentants des banques alimentaires et permettant de mieux soutenir les pratiques alimentaires sécuritaires en fonction des réalités des organismes qui dépendent des dons de nourriture pour fonctionner, et dont l'objectif est d'offrir des aliments nutritifs aux personnes dans le besoin, tout en tenant compte de la grande vulnérabilité de certaines sous-populations de clients (p. ex. les aînés, les enfants, les personnes immunodéprimées) aux agents pathogènes d'origine alimentaire ${ }^{28}$.

Les participants à cette étude ont pu discuter des lois, des règlements et des politiques relatifs à la salubrité des aliments, qui était beaucoup plus clairs que ceux associés à la sécurité alimentaire, en partie parce que les lois sur la salubrité des aliments existent depuis beaucoup longtemps au Canada (p. ex. la Loi sur les aliments et drogues $^{29}$, une loi canadienne qui remonte à 1920) que les lois sur la sécurité alimentaire, relativement nouvelles (p. ex. la Food Donor Encouragement Act, $1997^{27}$ de la Colombie-Britannique et le projet de loi M 222 sur les aliments locaux [BC Local Food $\left.\mathrm{Act}^{30}\right]$ ). Cela peut exacerber les conflits entre les praticiens des deux secteurs, pour qui les lois peuvent limiter les activités de santé publique. Dans notre étude, si les participants ont fait état des conséquences positives et négatives de la Food Safety $A t^{31}$ de la Colombie-Britannique dans le cadre de leurs pratiques quotidiennes (surtout celles liées à l'application des normes de salubrité des aliments), d'autres lois n'ont pas été mentionnées aussi explicitement ni largement. La salubrité des aliments et la sécurité alimentaire constituent 2 des 21 programmes de santé publique de base de la province, et leur interdépendance est de plus en plus reconnue $^{32}$, l'accès à des aliments salubres étant considéré comme une constituante importante de la sécurité alimentaire. Le programme de base de salubrité des aliments est axé sur la réduction des préjudices liés aux contaminants microbiens et chimiques possibles et repose sur deux lois provinciales : la Food Safety Act $^{31}$ et la Public Health $A c t^{33,34}$. Le programme de sécurité alimentaire de base, qui vise à jeter les bases d'une saine alimentation et d'un approvisionnement alimentaire stable et durable ${ }^{35}$, est également soutenu par la Food Safety Act et la Public Health Act ${ }^{36}$, de même que par la Food Donor Encouragement Act ${ }^{27,36}$. Les fondements législatifs communs à ces programmes de base laissent penser que ce n'est peut-être pas la législation comme telle mais plutôt son interprétation et son application (notamment par l'entremise de politiques et de pratiques qui souvent ne tiennent compte que d'un seul effet sur l'alimentation ou la santé) qui peuvent créer des tensions dans la pratique en santé publique. En effet, certains participants ont expliqué comment le fait de réinterpréter certains règlements existants peut contribuer à renforcer à la fois les objectifs de salubrité des aliments et ceux de sécurité alimentaire. Examiner comment les lois actuelles pourraient être réinterprétées est important, mais cela dépasse le cadre de notre étude.

Les participants ont parlé des aliments spécifiques qui comportent à la fois des risques et des avantages pour la santé, dont les aliments transformés et emballés ainsi que les fruits et légumes frais ont constitué les exemples prédominants. Comme les fruits et légumes frais sont beaucoup à l'origine de maladies d'origine alimentaire au Canada ${ }^{10}$, des interventions sur l'environnement alimentaire destinées à accrồtre l'accès aux fruits et légumes frais et leur disponibilité devraient être menées de façon proactive pour atténuer le risque d'exposition aux agents pathogènes. Jusqu'à maintenant, l'écosystème microbien dynamique des aliments n'a pas été explicitement considéré comme une composante d'un environnement alimentaire sain, ce qui devrait l'être dans l'avenir. Pour ce faire, d'après nos résultats, au moment de caractériser un environnement alimentaire, on devrait mesurer les facteurs de salubrité des aliments. À ce jour, les études sur l'environnement alimentaire canadien ayant tenu compte de la qualité et de la salubrité 
des aliments ont porté sur des composantes comme la fraîcheur perçue ${ }^{37}$ et la sécurité physique relative à l'acheminement des produits vers les établissements alimentaires $^{38}$, et ont fait ressortir des problèmes liés à la contamination d'aliments par des souris $^{39}$. Malgré les données probantes américaines selon lesquelles les aliments, particulièrement les fruits et légumes frais, provenant de marchés et d'établissements de vente au détail situés dans des régions désavantagées sur le plan socioéconomique peuvent présenter des taux de contamination microbienne plus élevés que dans des régions à statut socioéconomique élevé $\mathrm{u}^{40-42}$, ces indicateurs de salubrité n'ont pas été inclus dans les évaluations de l'environnement alimentaire canadien. L'une des raisons invoquées pour expliquer cette omission est le manque de données ${ }^{25}$, mais étant donné la quantité importante de données d'inspection recueillies en permanence par les organismes locaux et provinciaux de santé publique (p. ex. Vancouver Coastal Health ${ }^{43}$, Région de Waterloo $^{44}$ ), l'intégration de mesures de la salubrité des aliments dans la caractérisation de l'environnement alimentaire est en principe réalisable et devrait être étudiée plus à fond.

D'autres recherches ayant examiné le lien entre salubrité des aliments et sécurité alimentaire portaient sur l'évaluation de l'inclusion de la salubrité des aliments dans les initiatives de sécurité alimentaire ${ }^{45}$ et les répercussions d'une réglementation particulière en matière de salubrité sur la sécurité alimentaire de la population ${ }^{11,12}$, de même que sur l'examen des facteurs de risque qui peuvent être communs à l'insécurité alimentaire et aux maladies d'origine alimentaire (p. ex. le statut socioéconomique $^{46}$, le changement climatique $^{47}$ ). Notre étude renforce les résultats de ces travaux, en définissant de multiples échelles où se recoupent la salubrité des aliments et la sécurité alimentaire (c.-à-d. les produits alimentaires, les pratiques de santé publique, les politiques gouvernementales, etc.) Elle permet également de souligner les problèmes présents dans le système alimentaire canadien, à savoir la séparation entre salubrité des aliments et sécurité alimentaire dans la pratique en santé publique, et le degré relativement plus élevé d'institutionnalisation du rôle de la salubrité des aliments en santé publique par rapport à celui de la sécurité alimentaire. Dans notre étude, les praticiens en salubrité des aliments occupaient des postes clairement définis, dont le poste accrédité d'agent d'hygiène du milieu ${ }^{48}$, tandis que le rôle des praticiens en sécurité alimentaire était plus diversifié et englobait souvent les nutritionnistes communautaires et les diététistes en santé publique. Les praticiens en salubrité des aliments travaillaient uniquement dans les organismes gouvernementaux et les autorités sanitaires, alors qu'on trouvait des praticiens en sécurité alimentaire également dans les organismes communautaires et les organisations non gouvernementales. Ce facteur peut être important lorsqu'on envisage la création d'initiatives alimentaires axées sur la mobilisation de la communauté, parce que les organismes communautaires pourraient ne pas représenter ni défendre les intérêts liés à la prise en compte des risques réels d'origine alimentaire auxquelles les Canadiens sont confrontés. Par exemple, au moment de la rédaction de cet article, la ColombieBritannique disposait de nombreux réseaux communautaires de sécurité alimentaire, dont 14 dans la région de Vancouver seulement ${ }^{49}$, mais aucun groupe communautaire de promotion de la salubrité des aliments. Les projets liés à la santé publique, comme l'élaboration de politiques en matière d'aliments locaux, qui font entendre la voix des communautés dans les discussions, sont susceptibles de ne pas totalement régler les problèmes de salubrité des aliments dans leurs activités. Si cela a des répercussions sur la salubrité des aliments, risques auxquels les praticiens de ce domaine doivent répondre d'une façon qui est perçue comme négative (p. ex. fermeture des locaux, rappel d'aliments), un cycle de désengagement et de méfiance est possible, comme l'ont mentionné dans l'étude les participants du secteur de la salubrité des aliments. La mobilisation des différents secteurs dès la mise en place de mesures de santé publique peut constituer un excellent moyen de réduire cette division.

\section{Points forts et limites}

La littérature sur le sujet étant pauvre, nous avons pu, grâce à des entrevues menées auprès des informateurs clés, procéder à un examen approfondi des modalités dont les secteurs de la sécurité alimentaire et de la salubrité des aliments peuvent se recouper, à partir de l'expérience de praticiens en santé publique de la ColombieBritannique, révélant ainsi plusieurs éléments importants à prendre en compte lors de la conception de l'environnement alimentaire ou de la mise en œuvre de mesures visant à le transformer. Nos travaux peuvent fournir une base à des évaluations futures plus exhaustives, portant sur un éventail plus vaste de praticiens et de provinces. La principale limite de notre étude réside dans le fait que nous avons ciblé des personnes possédant une expérience de travail avec l'autre secteur : il est possible que leur expérience diffère de celle d'autres personnes n'ayant jamais travaillé avec l'autre secteur ou ayant essayé mais en vain. De même, nos participants travaillaient tous en santé publique, de sorte que les tensions et les recoupements signalés ici ne sont peut-être pas représentatifs ce qui est vécu hors du domaine de la santé publique. Des entrevues avec d'autres personnes participant à l'amélioration de la salubrité des aliments et de la sécurité alimentaire (p. ex. enseignants des compétences alimentaires, exploitants de soupes populaires) sont nécessaires pour mieux mettre au jour les tensions et les considérations au carrefour de ces deux secteurs, au-delà du cadre de la santé publique. Cette étude a néanmoins fait ressortir d'importants éléments à prendre en considération lors de l'élaboration d'activités et de politiques en matière de santé publique contribuant à façonner l'environnement alimentaire canadien.

\section{Conclusion}

Notre étude met en évidence comment la sécurité alimentaire et la salubrité des aliments, deux secteurs de la santé publique importants mais historiquement séparés au Canada, sont en fait liées de plusieurs manières. Elle démontre globalement qu'il faut compter les maladies d'origine alimentaire comme les activités liées à la salubrité des aliments au nombre des facteurs importants qui ont une incidence sur un environnement alimentaire sain au Canada. Il incombe aux praticiens œuvrant dans ces secteurs de collaborer davantage, en particulier pour atténuer les conséquences non prévues sur la santé publique des activités visant à accroître l'accès aux aliments frais et leur disponibilité (dont les fruits et légumes frais) ou à limiter l'exposition aux aliments qui présentent un risque microbien élevé. D'après nos résultats, au-delà de la sécurité alimentaire et de la salubrité des aliments, il importe d'examiner comment les diverses mesures en santé publique relatives à l'alimentation et à la santé sont susceptibles d'interférer de manière non prévue pour façonner l'environnement alimentaire actuel, soulignant ainsi l'importance de mobiliser les 
différents secteurs au sein des organismes de santé publique et entre eux lorsqu'il s'agit de concevoir des programmes ou des politiques visant à transformer les habitudes alimentaires des Canadiens.

\section{Remerciements}

Le financement des entrevues auprès d’informateurs clés a été assuré par un contrat du Centre de contrôle des maladies de la Colombie-Britannique (BCCDC). Les auteurs remercient Karen Rideout et Melanie Kurrein (BCCDC) pour leur soutien lors de ces travaux. Les opinions exprimées dans ce document sont celles des auteurs et non celles du BCCDC. Les auteurs remercient également Abra Brynne (BC Food Systems Network) pour la discussion autour des concepts mentionnés dans ce document et pour sa rétroaction à ce propos.

\section{Conflits d'intérêts}

Tous les auteurs déclarent qu'ils ne font l'objet d'aucun conflit d'intérêts ou d'intérêts concurrentiels.

\section{Contributions des auteurs}

SM a conçu l'étude, KS a analysé les données avec la participation de tous les auteurs, tous les auteurs ont interprété les données, KS et SM ont rédigé le manuscrit et tous les auteurs ont revu et approuvé la version définitive.

\section{Références}

1. Rideout K, Mah CL, Minaker L. Environnements alimentaires : Introduction pour professionnels de la santé publique [Internet]. Vancouver (C.-B.), Centre de collaboration nationale en santé environnementale; 2015. En ligne à : http://www.ccnse.ca/sites /default/files/Environnements_ alimentaires_professionnels_sante_ publique_dec_2015.pdf

2. Organisation des Nations Unies pour l'alimentation et l'agriculture. Déclaration de Rome sur la sécurité alimentaire mondiale et Plan d'action sur le Sommet mondial de l'alimentation [Internet]. Rome (IT): FAO; 1996 [consultation le 4 avril 2016]. En ligne à : http://www.fao.org/docrep/003 /W3613F/W3613F00.HTM
3. BC Association of Farmers' Markets. Farmers' Market Nutrition Coupon Program [Internet]. Vancouver (BC): BC Association of Farmers' Markets; 2014 [consultation le 4 avril 2016]. En ligne à : http://www.bcfarmersmarket .org/nutrition-coupon-program

4. Government of British Columbia. Food security [Internet]. Vancouver (BC): Government of British Columbia; 2017 [consultation le 12 avril 2017]. En ligne à : http://www2.gov.bc.ca/gov /content/health/keeping-bc-healthy -safe/food-safety/food-safety-sanitation -plans/food-security

5. Loi sur la salubrité des aliments au Canada, L.C. 2012, ch. 24 [Internet]. Ottawa (Ont.) : ministre de la Justice; [modification le 19 juin 2014]. En ligne à : http://laws-lois.justice.gc.ca /PDF/S-1.1.pdf

6. McIntyre L, Wilcott L, Naus M. Listeriosis outbreaks in British Columbia, Canada, caused by soft ripened cheese contaminated from environmental sources. BioMed Res Int. 2015;131623. doi: 10.1155/2015/131623.

7. MacDonald DM, Fyfe M, Paccagnella A, Trinidad A, Louie K, Patrick D. Escherichia coli O157:H7 outbreak linked to salami, British Columbia, Canada, 1999. Epidemiol Infect. 2004;132: 283-289. doi: 10.1017/S0950268803001651.

8. Majowicz SE, Meyer SB, Kirkpatrick SI, et al. Food, health, and complexity: towards a conceptual understanding to guide collaborative public health action. BMC Public Health. 2016;16: 487. doi: 10.1186/s12889-016-3142-6.

9. Painter JA, Hoekstra RM, Ayers T, et al. Attribution of foodborne illnesses, hospitalizations, and deaths to food commodities by using outbreak data, United States, 1998-2008. Emerg Infect Dis. 2013;19(3):407-415. doi: 10 $.3201 /$ eid1903.111866.

10. Kozak GK, MacDonald D, Landry L, Farber JM. Foodborne outbreaks in Canada linked to produce: 2001 through 2009. J Food Prot. 2013;76(1): 173-183. doi: 10.4315/0362-028X.JFP $-12-126$.
11. Miewald C, Hodgson S, Ostry A. Tracing the unintended consequences of food safety regulations for community food security and sustainability: small-scale meat processing in British Columbia. Local Environ. 2015;20(2): 237-255. doi: 10.1080/13549839.2013 .840567 .

12. Miewald C, Ostry A, Hodgson S. Food safety at the small scale: the case of meat inspection regulations in British Columbia's rural and remote communities. J Rural Studies. 2013;32:93-102. doi: 10.1016/j.jrurstud.2013.04.010.

13. Minaker LM, Shuh A, Olstad DL, Engler-Stringer R, Black JL, Mah CL. Retail food environments research in Canada: a scoping review. Can J Public Health. 2016;107(suppl. 1):eS4eS13. doi: 10.17269/CJPH.107.5344.

14. Glanz K, Sallis JF, Saelens BE, Frank LD. Healthy nutrition environments: concepts and measures. Am J Health Promot. 2005;19(5):330-333.

15. Vine MM, Elliott SJ. Examining locallevel factors shaping school nutrition policy implementation in Ontario, Canada. Public Health Nutr. 2014;17(6): 1290-8. doi: 10.1017/S1368980013002516.

16. Cavanaugh E, Green S, Mallya G, Tierney A, Brensinger C, Glanz K. Changes in food and beverage environments after an urban corner store intervention. Prev Med. 2014;65:7-12. doi: 10.1016/j.ypmed.2014.04.009.

17. Mah CL, Cook B, Rideout K, Minaker LM. Policy options for healthier retail food environments in city-regions. Can J Public Health. 2016;107(suppl. 1):eS64-eS67. doi: 10.17269/cjph.107 .5343 .

18. Braun V, Clarke V. Using thematic analysis in psychology. Qual Res Psychol. 2006;3(2):77-101. doi: 10.1191 /1478088706qp063oa.

19. Morse JM, Barrett M, Mayan M, Olson K, Spiers J. Verification strategies for establishing reliability and validity in qualitative research. Int $\mathrm{J}$ Qual Methods. 2002;1(2):13-22.

20. Sandelowski M. Focus on research methods: whatever happened to qualitative description? Res Nurs Health. 2000;23(4):334-340. 
21. Birks M, Chapman Y, Francis K. Memoing in qualitative research. J Res Nurs. 2008;13(1):68-75. doi: 10.1177 /1744987107081254.

22. Province of British Columbia. FOODSAFE [Internet]. Vancouver (BC): Province of British Columbia; 2009 [consultation le 20 octobre 2016]. En ligne à : http://www.foodsafe.ca

23. Thomas MK, Murray R, Flockhart L, et al. Estimates of the burden of foodborne illness in Canada for 30 specified pathogens and unspecified agents, circa 2006. Foodborne Pathog Dis. 2013;10(7):639-648. doi: 10.1089/fpd .2012 .1389 .

24. Community Care and Assisted Living Act: Child Care Licensing Regulation, Reg. 332; 2007. En ligne à : http:// www.bclaws.ca/civix/document/id /complete/statreg/332_2007

25. Santé Canada. Mesure de l'environnement alimentaire au Canada. Ottawa (Ont.) : ministre de la Santé; 2013. [n ${ }^{\circ}$ de cat. : H164-155/2012E-PDF]. 98 p.

26. Martin W, Perkin K. Food safety and food security: mapping relationships. J Agric Food Syst Community Dev. 2016;6(2):13-24. doi: http://dx.doi.org /10.5304/jafscd.2016.062.001

27. Food Donor Encouragement Act (SBC 1997) - Chapter 8. Victoria (BC): Queen's Printer; 1997. En ligne à : http: / / ww w.bclaws.ca/Recon /document/ID/freeside/00_97008_01

28. BC Centre for Disease Control, Greater Vancouver Food Bank, Food Banks BC. Guidelines for food distribution organizations with grocery or meal programs [Internet]. Vancouver (BC): BC Centre for Disease Control, Environmental Health Services; février 2016 [consultation le 13 avril 2017]. En ligne à : www.bccdc.ca/health -info/food-your-health/healthy-food -access-food-security

29. Loi sur les aliments et drogues, LRC (1985), ch. F-27. Ottawa (Ont.) : ministre de la Justice; 2016 [mise à jour le 12 décembre 2016]. En ligne à : http://laws-lois.justice.gc.ca/PDF/F -27.pdf
30. Bill M 222-2015 British Columbia Local Food Act, 2015. Victoria (BC): Queen's Printer; 2015. En ligne à : http://www.bclaws.ca/civix/document /id/lc/billsprevious/4th40th:m222-1

31. Food Safety Act (SBC 2002) - Chapter 28. Victoria (BC): Queen's Printer; 2002. En ligne à : http://www.bclaws .c a / E P Libraries / b claws_new /document/ID/freeside/00_02028_01

32. Provincial Health Services Authority. 2008-09 Annual progress update: core public health programs [Internet]. 2009 [consultation le 27 octobre 2016]. En ligne à : http://www.phsa.ca/ Documents/200809corepublichealth programsprogressupdate.pdf

33. Public Health Act (SBC 2008) - Chapter 28. Victoria (BC): Queen's Printer; 2008. En ligne à : http://www.bclaws .ca/civix/document/id/complete /statreg/08028_01

34. Government of British Columbia. Food safety legislation [Internet]. n.d. [consultation le 16 juin 2017]. Consultable en ligne à la page : http://www2.gov .bc.ca/gov/content/health/keeping-bc -healthy-safe/food-safety/food-safety -legislation

35. Government of British Columbia. Food security [Internet]. Victoria (BC): Government of British Columbia; [date inconnue; consultation le 16 juin 2017]. En ligne à : http://www2.gov .bc.ca/gov/content/health/keeping -bc-healthy-safe/food-safety/food -safety-sanitation-plans/food-security

36. BC Ministry of Health. Model core program paper: food security. Victoria (BC): BC Ministry of Health; 2006 [mise à jour : mars 2014]. En ligne à : http://www2.gov.bc.ca/assets/gov /health/about-bc-s-health-care-system /public-health/healthy-living-and -healthy-communities/food_security _model_core_program_paper.pdf

37. Lebel A, Noreau D, Tremblay L, et al. Identifying rural food deserts: methodological considerations for food environment interventions. Can J Public Health. 2016; 107 (suppl. 1):eS21-eS26. doi: 10.17269/CJPH.107.5353.
38. Le H, Engler-Stringer R, Muhajarine $\mathrm{N}$. Walkable home neighbourhood food environment and children's overweight and obesity: proximity, density or price? Can J Public Health. 2016; 107(suppl. 1): eS42-eS47. doi: 10.17269 /CJPH.107.5347.

39. DyckFehderau D, Holt NL, Ball GD, Alexander First Nation Community, Willows ND. Feasibility study of asset mapping with children: identifying how the community environment shapes activity and food choices in Alexander First Nation. Rural Remote Health. 2013;13(2):2289.

40. Koro ME, Anandan S, Quinlan JJ. Microbial quality of food available to populations of differing socioeconomic status. Am J Prev Med. 2010; 38(5):478-481.

41. Signs RJ, Darcey VL, Carney TA, Evans AA, Quinlan JJ. Retail food safety risks for populations of different races, ethnicities, and income levels. J Food Prot. 2011;74(10):1717-1723. doi: 10.4315/0362-028X.JFP-11-059.

42. Quinlan JJ. Foodborne illness incidence rates and food safety risks for populations of low socioeconomic status and minority race/ethnicity: a review of the literature. Int $\mathrm{J}$ Environ Res Public Health. 2013;10(8):36343652. doi: 10.3390/ijerph10083634

43. Vancouver Coastal Health. Food premises [Internet]. Vancouver (BC): Vancouver Coastal Health; [date inconnue; consultation le 26 janvier 2017]. En ligne à : https://inspections .vcha.ca

44. Region of Waterloo. Public Health Inspection Results [Internet]. Waterloo (Ont.) : Region of Waterloo; 2010. En ligne à : http://www.regionofwaterloo .ca/en/safeHealthyCommunity/Public -Health-Inspection-Results.asp

45. Dollahite JS, Pijai EI, Scott-Pierce M, Parker C, Trochim W. A randomized controlled trial of a community-based nutrition education program for lowincome parents. J Nutr Educ Behav. 2014;46(2):102-109. doi: 10.1016/j.jneb .2013.09.004. 
46. Gillespie IA, Mook P, Little CL, Grant KA, McLauchlin J. Human listeriosis in England, 2001-2007: association with neighbourhood deprivation. Euro Surveill. 2010;15(27):7-16.

47. Ahdoot S, Pacheco SE, The Council on Environmental Health. Global climate change and children's health. Pediatrics. 2015;136(5):e1-e17. doi: 10.1542/peds .2015-3233

48. Province of British Columbia. Ministerial Order No. M217. Victoria (BC): Province of British Columbia; 2013. En ligne à : http://www.bclaws.ca/civix /document/id/mo/mo/2013_m217 /search/CIVIX_DOCUMENT_ROOT_ STEM:(environmental \% 20health $\% 20$ officer)? 1\#hit1

49. BC Food Security Gateway. Food security networks, policy councils, and agencies [Internet]. Vancouver (BC): Provincial Health Services Authority and the Public Health Association of BC; 2017. En ligne à : http://bcfood securitygateway.ca/about-bc-food -security-gateway/key-food-security -agencies/ 